\title{
Check-list de Lauraceae Juss. para Mato Grosso do Sul, Brasil
}

\author{
Flávio Macedo Alves', Daniel Quedes Domingos², Priscila Passala Vaz', \\ Rosani do Carmo de Oliveira Arruda' \& Vinicius Castro Souza ${ }^{3}$
}

\author{
'Universidade Federal de Mato Grosso do Sul, Centro de Ciências Biológicas e da Saúde, Botânica, CEP 79070-900. Campo Grande, MS \\ Brasil. flaurace@yahoo.com.br \\ 2Universidade Federal de Lavras, Departamento de Biologia, Campus Universitário \\ CEP 35200-000. Lavras, MG, Brasil. quedesbiologo@gmail.com \\ 3Universidade de São Paulo - Escola Superior de Agricultura "Luiz de Queiroz", Herbário ESA, Av. Pádua Dias 11, Caixa Postal 9 \\ CEP 13418-900, Piracicaba, SP, Brasil.
}

RESUMO - Lauraceae é um importante táxon componente da estrutura florística de grande parte dos ecossistemas florestais do Brasil. Por essa razão, o presente trabalho apresenta o checklist desta família para o Mato Grosso do Sul, com o objetivo de contribuir para o conhecimento da flora do estado, ainda pouco estudada, bem como para distribuição geográfica de Lauraceae no Brasil. O trabalho foi baseado em análise de exsicatas de coleções botânicas nacionais e estrangeiras, além de inúmeras coletas no estado. Foram registradas 27 espécies subordinadas a 10 gêneros: Aiouea Aubl., Aniba Aubl., Cassytha L., Cinnamomum Schaeff., Endlicheria Nees, Licaria Aubl., Mezilaurus Taub., Nectandra Rol. ex Rottb., Ocotea Aubl. e Persea Mill.

Palavras-chave: biodiversidade, Cerrado, Pantanal.

ABSTRACT - Checklist of the Lauraceae Juss. from Mato Grosso do Sul, Brazil. Lauraceae is an important component of the floristic structure of great part of Brazilian ecosystems. Therefore, this study present a checklist of Lauraceae in Mato Grosso do Sul, based on national and foreign herbaria and collecting in several areas at state. In this survey, 27 species belonging to 10 genera:Aiouea Aubl., Aniba Aubl., Cassytha L., Cinnamomum Schaeff., Endlicheria Nees, Licaria Aubl., Mezilaurus Taub., Nectandra Rol. ex Rottb., Ocotea Aubl. and Persea Mill, were identified.

Keywords: biodiversity, Cerrado, Pantanal.

\section{INTRODUÇÃO}

Lauraceae é uma família de árvores e arbustos, com a exceção de Cassytha L., uma herbácea parasita. A família possui distribuição predominantemente tropical, especialmente diversificada no sudeste da Ásia e norte da América do Sul, com poucos representantes em regiões temperadas. A família é composta por aproximadamente 3000 espécies incluídas em 52 gêneros (Rohwer 1993a). Nas Américas ocorrem cerca de 30 gêneros e 1000 espécies de Lauraceae com grande diversidade na América do Sul e América Central (Madriñán 2004). No Brasil a família está representada por 23 gêneros e cerca de 420 espécies (Barroso et. al. 2002, Quinet et al. 2010).

Lauraceae está entre as famílias que mais contribuem para a riqueza da flora nos neotrópicos (Gentry 1988, Kubitzki \& Kurz 1984). Em termos florísticos e econômicos destaca-se como uma das mais importantes famílias de Angiospermas (Burger 1988, van der Werff 1991, Rohwer et al. 1991, Baitello 2001, Caiafa \& Martins 2007), sendo a quarta maior família de plantas arbóreas do mundo (Beech et al. 2017, no prelo). Lauraceae pode ser o principal componente em altitudes intermediárias dos Andes (Gentry 1988) e em florestas do sudeste asiático (Whitmore \&
Sidiyasa 1986) e a segunda principal família arbórea na Floresta Ombrófila Densa Atlântica no Brasil (Lima et al. 2012). No Brasil, Lauraceae representa uma das famílias de maior destaque na composição florística de grande parte dos ecossistemas florestais na Mata Atlântica e Amazônia (Souza \& Lorenzi 2008).

A família possui relevante importância econômica destacando-se espécies como o abacate (Persea americana Mill.), a canela (Cinnamomum zeylanicum Blume) e o louro (Laurus nobilis L.) utilizadas em todo o mundo na alimentação. Substâncias aromáticas são extraídas de algumas espécies, como a canela-sassafrás (Ocotea odorifera Rohwer) e o pau-rosa (Aniba rosaeodora Ducke). A madeira das Lauraceae (Ocotea, Nectandra e Mezilaurus) é amplamente explorada em diversas regiões para diferentes finalidades devido a sua alta durabilidade e resistência (Vicentini et al. 1999).

Lauraceae possui reputação de ser uma das famílias de Angiospermas, na região neotropical, mais difíceis para identificação e apresenta grande número de espécimes citados sem identificação específica em estudos florísticos (Caiafa \& Martins 2007, Thomaz \& Monteiro 1997). Além do mais, espécies da família não podem ser identificadas seguramente baseadas apenas em caracteres vegetativos 
(Rohwer 1993a), uma vez que chaves de identificação e estudos taxonômicos estão fundamentados em atributos florais (van der Werff 1991).

No Brasil, destacam-se os trabalhos de taxonomia com Lauraceae de Meissner (1866), Sampaio (1917), Barroso (1949), Vattimo-Gil (1956a, b, 1957, 1958, 1959, 1966a, b, 1976, 1978a, b, 1979a, b, c, 1980a, b), Coe-Teixeira (1963, 1965, 1975, 1980), Pedralli (1984, 1986, 1987) e Baitello \& Coe-Teixeira (1987); mais recentemente, os de Vicentini et al. (1999), Quinet \& Andreata (2002), Baitello et al. (2003), Assis et al. (2005), Quinet (2005), Kropf et al. (2006) e Moraes (2005, 2006); e as listagens da Flora da Mata Atlântica de Quinet (2009) e da Flora do Brasil por Quinet et al. (2010). Para Mato Grosso do Sul foram realizados apenas a listagem de Dubs (1998) e os estudos taxonômicos para o município de Corumbá (Alves \& Ishii 2007) e Nectandra Rol. ex Rottb. para todo o estado (Alves \& Sartori 2009).

Mato Grosso do Sul, apesar de reunir elementos fitogeográficos heterogêneos que tornam sua vegetação interessante para estudos florísticos e biogeográficos, incluindo os biomas Pantanal, Cerrado, Mata Atlântica e Chaco (Rizzini 1979), apresenta uma flora pouco conhecida (Pott et al. 2006), sendo um dos estados brasileiros com menores índices de coletas botânicas por quilômetro quadrado (Peixoto 2003, Martinelli \& Martins 2010). A intensa atividade agrícola, com a abertura de novas fronteiras para o cultivo de grãos e pastagens, tem levado a uma crescente degradação das florestas e campos nativos, para os quais estratégias de reflorestamento serão imprescindíveis. Por essa razão, lacunas no conhecimento florístico podem comprometer a elaboração de estratégias para conservação (Souza 2010).

Sendo assim, o presente trabalho teve como objetivo apresentar um checklist de Lauraceae para o estado de Mato Grosso do Sul contribuindo para o conhecimento da flora do estado, bem como para o conhecimento da distribuição geográfica da família no Brasil.

\section{MATERIAL E MÉTODOS}

Para a realização do presente estudo foram examinadas exsicatas pertencentes a herbários nacionais e estrangeiros indexados (BHCB, CGMS, COR, CPAP, ESA, FUEL, HB, HRCB, HUFU, IAC, INPA, JBRJ, MBM, NY, PAMG, RB, SP, SPF, SPSF, UB, UEC, UFMT e UPCB) (siglas segundo Holmgren et al. 1990) e quatro não indexados (CEUL, DDMS, HISA e HSJRP). Coletas foram realizadas em diferentes regiões do Mato Grosso do Sul, sendo os espécimes herborizados e incorporados aos herbários CGMS e COR. Os mapas foram confeccionados no programa ArcMap, tendo o mapa de esforço de coleta sido baseado no número de exsicatas de Lauraceae amostradas no estado, proveniente dos herbários citados.

\section{RESULTADOS E DISCUSSÃO}

Em Mato Grosso do Sul foram encontradas 27 espécies de Lauraceae, subordinadas a 10 gêneros, sendo Nectandra
Rol. ex Rottb. e Ocotea Aubl., ambos com oito espécies os gêneros mais ricos, seguidos de Endlicheria Nees e Mezilaurus Taub. com duas espécies cada. Aiouea Aubl., Aniba Aubl., Cassytha L., Cinnamomum Schaeff., Licaria Aubl. e Persea Mill. foram representados apenas por uma espécie (Quadro 1).

Com relação às listagens de Lauraceae disponibilizadas para Mato Grosso do Sul (Quadro 2), Dubs (1998) citou 20 espécies subordinadas a cinco gêneros, sendo Ocotea com 12 espécies e Nectandra com cinco, os mais representativos, seguidos de Aiouea, Cassytha e Endlicheria com apenas uma espécie cada. Na Lista de Espécies da Flora do Brasil, Quinet et al. (2012) relataram sete gêneros e 30 espécies de Lauraceae para Mato Grosso do Sul. De acordo com os autores citados, os gêneros mais representativos foram Ocotea com 12 espécies, Nectandra com sete e Mezilaurus com quatro. No tocante à diversidade genérica, Nectandra e Ocotea representaram cerca de 60\% das Lauraceae de Mato Grosso do Sul. Esse resultado também refletiu a diversidade genérica da família no Brasil, pois, Ocotea (155) e Nectandra (46) são, realmente, os gêneros mais ricos (Quinet et al. 2012) e apresentam aproximadamente $50 \%$ da riqueza de Lauraceae, resultado confirmado em outros estados no Brasil, assim como para o Rio de Janeiro (Quinet 2005), Goiás e Tocantins (Moraes 2005) e São Paulo (Baitello et al. 2003).

$\mathrm{Na}$ Lista de Espécies da Flora do Brasil, Quinet et al. (2010) citaram a ocorrência de apenas uma espécie de Endlicheria para Mato Grosso do Sul. No entanto, foram encontradas no presente trabalho Endlicheria paniculata (Spreng.) J.F. Macbr. e Endlicheria lhotzkyi (Nees) Mez. Por outro lado, quatro espécies de Mezilaurus foram mencionadas para o estado, entretanto, apenas as duas características do cerrado brasileiro (Alves \& Baitello 2008, van der Werff 1987, Alves 2011) foram confirmadas. Aiouea e Aniba foram representados na Lista de Espécies da Flora do Brasil (Quinet et al. 2010) por três e duas espécies, respectivamente. Todavia, no presente trabalho, apenas Aiouea trinervis Meisn. e Aniba heringeri Vatt. foram ratificadas.

Aiouea trinervis, Cassytha filiformis L., Nectandra cissiflora Nees, Nectandra gardneri Meisn., Nectandra hihua (Ruiz \& Pav.) Rohwer, Nectandra megapotamica (Spreng.) Mez, Ocotea corymbosa (Meisn.) Mez, Ocotea densiflora (Meisn.) Mez, Ocotea diospyrifolia (Meisn.) Mez, Ocotea lancifolia (Schott) Mez, Ocotea minarum (Nees \& Mart.) Mez e Ocotea velloziana Mez foram mencionadas em todas as listagens de Lauraceae para Mato Grosso do Sul (Quadro 2). Por outro lado, os gêneros Cinnamomum, Licaria e Persea que não haviam sido citados em levantamentos anteriores de Lauraceae para o estado, foram confirmados no presente trabalho com uma espécie cada (Quadro 2).

A maior parte das espécies de Lauraceae encontradas em Mato Grosso do Sul apresenta ampla distribuição no território brasileiro. Entre as espécies com ampla distribuição destacam-se Aiouea trinervis, Cassytha 
Quadro 1. Espécies de Lauraceae no Mato Grosso do Sul. *Espécies citadas na lista de espécies ameaçadas da IUCN (IUCN 2001). **Espécie citada como ameaçada tanto na Lista de Espécies Ameaçadas da IUCN (IUCN 2001) quanto na Lista de Espécies Ameaçadas de Extinção da Flora Brasileira do Ministério do Meio Ambiente (MMA). Categorias da IUCN (IUCN 2001): EN = Em Perigo; VU = Vulnerável; LR = Baixo Risco.

\begin{tabular}{|c|c|c|}
\hline Espécies & Voucher & Formação Vegetacional \\
\hline Aiouea trinervis Meisn. & U.M. Rezende 181 (CGMS) & Cerrado, Mata Ciliar e Floresta Estacional Semidecidual \\
\hline Aniba heringeri Vatt. & G.A. Damasceno-Júnior et al. 3025 (CGMS) & Cerrado, Matas Ciliares \\
\hline Cassytha filiformis L. & U.M. Rezende et al. 210 (CGMS) & Cerrado e Pantanal \\
\hline $\begin{array}{l}\text { Cinnamomum triplinerve (Ruiz \& Pav.) } \\
\text { Kosterm. }\end{array}$ & W. Garcez 196 (CGMS) & Floresta Estacional Semidecidual \\
\hline Endlicheria paniculata (Spreng.) J.F.Macbr. & L.C. Rodrigues 92 (CGMS) & Cerrado, Matas Ciliares e Floresta Estacional Semidecidual \\
\hline E. lhotzkyi (Nees) Mez & G. Frison s.n. (CGMS, ESA) & Cerrado, Mata Ciliar \\
\hline Licaria sp. & $F-49$ (CGMS 15438) & Floresta Estacional Semidecidual \\
\hline $\begin{array}{l}\text { Mezilaurus crassiramea (Meisn.) Taub. } \\
\text { ex Mez }\end{array}$ & F. M. Alves 530 (CGMS, ESA, SPF) & Cerrado \\
\hline M. vanderwerffii F.M. Alves \& J.B. Baitello & F. M. Alves \& W. Garcez 45 (CGMS, ESA) & Cerrado e Floresta Estacional Semidecidual \\
\hline Nectandra amazonum Nees & G.A. Damasceno-Júnior 2304 (COR, SPF) & Pantanal, Mata Ciliar \\
\hline N. angustifolia (Schrad.) Nees & V. J. Pott \& A. Pott 4771 (CPAP) & Mata Ciliar e Floresta Estacional Semidecidual \\
\hline N. cissiflora Nees & G. A. Damasceno-Júnior 1547 (CGMS) & Cerrado, Mata Ciliar \\
\hline N. cuspidata Nees & C. A. Conceição 2008 (CGMS, RB) & Cerrado, Mata Ciliar \\
\hline N. gardneri Meisn. & A. Pott \& V. J. Pott 7770 (HMS) & Cerrado, Mata Ciliar \\
\hline N. hihua (Ruiz \& Pav.) Rohwer & C. A. Conceição 1578 (CGMS, COR, UB) & Cerrado, Mata Ciliar e Floresta Estacional Semidecidual \\
\hline N. megapotamica (Spreng.) Mez & F.M. Alves 23 (CGMS) & Cerrado, Mata Ciliar e Floresta Estacional Semidecidual \\
\hline N. psammophila Nees* (EN) & F.M. Alves et al. 51 (CGMS) & Mata Ciliar e Floresta Estacional Semidecidual \\
\hline Ocotea aciphylla (Nees) Mez* (LR) & U. Rezende \& F. Dias 1361 (CGMS) & Floresta Estacional Semidecidual \\
\hline O. catharinensis $\mathrm{Mez}^{* *}(\mathrm{VU})$ & A. Pott 14768 (CGMS, ESA) & Floresta Estacional Semidecidual \\
\hline O. corymbosa (Meisn.) Mez & C. A. Conceição 2030 (CGMS) & Cerrado, Mata Ciliar \\
\hline O. densiflora (Meisn.) Mez & J.L.G. Salvador et al. 88 (CGMS) & Cerrado, Mata Ciliar \\
\hline O. diospyrifolia (Meisn.) Mez & U. M. Resende et al. 489 (CGMS). & Pantanal, Mata Ciliar \\
\hline O. lancifolia (Schott) Mez & U.M. Coelho \& J.A.M. Coelho 1338(CGMS) & Cerrado, Matas Ciliar \\
\hline O. minarum (Nees \& Mart.) Mez & C. A. Conceição 2057 (CGMS) & Cerrado \\
\hline O. velloziana $\mathrm{Mez}$ & A. Pott 4678 (CPAP) & Cerrado \\
\hline Persea willdenovii Kosterm.* (LR) & M.L. Bueno 657 (CGMS) & Floresta Estacional Semidecidual \\
\hline
\end{tabular}

Quadro 2. Histórico de coletas e listagens de Lauraceae para Mato Grosso do Sul.

\begin{tabular}{|c|c|c|}
\hline Dubs (1998) & $\begin{array}{l}\text { Lista de Espécies da Flora do Brasil } \\
\text { (Quinet et al. 2012) }\end{array}$ & Presente trabalho \\
\hline Aiouea trinervis Meisn. & Aiouea impressa (Meisn.) Kosterm. & Aiouea trinervis Meisn. \\
\hline Cassytha filiformis L. & A. piauhyensis (Meisn.) Mez & Aniba heringeri Vatt. \\
\hline Endlicheria paniculata (Spreng.) J.F.Macbr. & A. trinervis Meisn. & Cassytha filiformis L. \\
\hline Nectandra cissiflora Nees & Aniba canelilla (Kunth) Mez & $\begin{array}{l}\text { Cinnamomum triplinerve } \\
\text { (Ruiz \& Pav.) Kosterm. }\end{array}$ \\
\hline N. cuspidata Nees & A. heringeri Vattimo-Gil & Endlicheria paniculata (Spreng.) J.F.Macbr. \\
\hline N. gardneri Meisn. & Cassytha filiformis L. & E. lhotzkyi (Nees) Mez \\
\hline N. hihua (Ruiz \& Pav.) Rohwer & Endlicheria paniculata (Spreng.) J.F.Macbr. & Licaria sp. \\
\hline N. megapotamica (Spreng.) Mez & $\begin{array}{l}\text { Mezilaurus crassiramea (Meisn.) } \\
\text { Taub. ex Mez }\end{array}$ & $\begin{array}{l}\text { Mezilaurus crassiramea } \\
\text { (Meisn.) Taub. ex Mez }\end{array}$ \\
\hline Ocotea corymbosa (Meisn.) Mez & M. itauba (Meisn.) Taub. ex Mez & M. vanderwerffii F.M. Alves \& J.B. Baitello \\
\hline O. daphnifolia (Meisn.) Mez & M. lindaviana Schwacke \& Mez & Nectandra amazonum Nees \\
\hline O. densiflora (Meisn.) Mez & M. vanderwerffii F.M.Alves \& Baitello & N. angustifolia (Schrad.) Nees \\
\hline O. diospyrifolia (Meisn.) Mez & Nectandra amazonum Nees & N. cissiflora $\mathrm{Nees}$ \\
\hline
\end{tabular}


Quadro 2. Continuação.

\begin{tabular}{|c|c|c|}
\hline O. gracilis (Meisn.) Mez & N. cissiflora Nees & N. cuspidata Nees \\
\hline O. gracilipes $\mathrm{Mez}$ & N. gardneri Meisn. & N. gardneri Meisn. \\
\hline O. lancifolia (Schott) $\mathrm{Mez}$ & N. globosa (Aubl.) Mez & N. hihua (Ruiz \& Pav.) Rohwer \\
\hline O. minarum (Nees \& Mart.) Mez & N. hihua (Ruiz \& Pav.) Rohwer & N. megapotamica (Spreng.) Mez \\
\hline O. pauciflora (Meisn.) Mez & N. lanceolata Nees & N. psammophila Nees \\
\hline O. spixiana (Nees) Mez & N. megapotamica (Spreng.) Mez & Ocotea aciphylla (Nees) Mez \\
\hline O. velloziana $\mathrm{Mez}$ & Ocotea acutifolia (Nees) Mez & O. catharinensis $\mathrm{Mez}$ \\
\hline \multirow[t]{10}{*}{ O. variabilis (Nees) Mez } & O. cеrnиа (Nees) Mez & O. corymbosa (Meisn.) Mez \\
\hline & O. corymbosa (Meisn.) Mez & O. densiflora (Meisn.) Mez \\
\hline & O. densiflora (Meisn.) Mez & O. diospyrifolia (Meisn.) Mez \\
\hline & O. diospyrifolia (Meisn.) Mez & O. lancifolia (Schott) Mez \\
\hline & O. glaziovii $\mathrm{Mez}$ & O. minarum (Nees \& Mart.) Mez \\
\hline & O. lancifolia (Schott) Mez & O. velloziana (Meisn.) Mez \\
\hline & O. minarum (Nees \& Mart.) Mez & Persea willdenovii Kosterm. \\
\hline & O. obliqua Vicent. & \\
\hline & O. spectabilis (Meisn.) Mez & \\
\hline & O. velloziana (Meisn.) Mez & \\
\hline Total: 20 espécies & 30 espécies & 26 espécies \\
\hline
\end{tabular}

filiformis, Endlicheria paniculata, Mezilaurus crassiramea (Meisn.) Taub. ex Mez, Ocotea corymbosa, O. velloziana e Persea willdenovii Kosterm.* (LR). Por outro lado, entre as espécies com distribuição mais restrita, podem ser citadas Endlicheria lhotzkyi, Mezilaurus vanderwerffii F.M. Alves \& J.B. Baitello, endêmicas da região centro-oeste do Brasil.

A ocorrência das espécies de Lauraceae de Mato Grosso do Sul reflete o mosaico de vegetação do estado, com espécies características dos domínios fitogeográficos da Amazônia (Nectandra amazonum Nees), Cerrado (Mezilaurus crassiramea, Endlicheria lhotzkyi (Nees) Mez), Mata Atlântica (Nectandra psammophila Nees* (EN), Ocotea aciphylla (Nees) Mez* (LR), Ocotea catharinensis $\mathrm{Mez}^{* *}$ (VU) e P. willdenovii) e das florestas meridionais (N. megapotamica). Essa padrão de distribuição pode estar associado principalmente à influência florística ou distribuição limítrofe desses domínios fitogeográficos aqui encontrados. No estado, as espécies de Lauraceae são encontradas com frequência nas matas ciliares e na floresta estacional semidecidual (Quadro 1). Ocotea aciphylla, O. catharinensis e $O$. puberula constituem citações novas para Mato Grosso do Sul.

Em Mato Grosso do Sul foram encontradas quatro espécies de Lauraceae ameaçadas de extinção em diferentes níveis, segundo os critérios da International Union for Conservation of Nature and Natural Resources (IUCN 2001) (Quadro 1). Dentre tais espécies, Ocotea catharinensis deve representar a maior preocupação, pois é citada como "Vulnerável" (VU) tanto na Lista de Espécies Ameaçadas da IUCN (IUCN 2001), quanto na Lista de Espécies Ameaçadas de Extinção da Flora Brasileira do Ministério do Meio Ambiente (MMA 2012). A madeira dessa Lauraceae apresenta excelente qualidade e tradicionalmente tem sido utilizada em marcenaria, construção civil e naval, para produção de vigas, tacos, mobiliário em geral, assoalhos, painéis, compensados e moirões (Carvalho 1994). Óleos essenciais produzidos em sua casca apresentam, dentre outros componentes, o linalol, utilizado em perfumaria na fabricação de cosméticos (Carvalho 1994). Segundo Baitello et al. (2003) a madeira de Ocotea catharinensis pode substituir a "imbuia" $(O$. porosa (Nees) Barroso) na confecção de móveis. Nectandra psammophila possui ampla distribuição na região sudeste do Brasil, porém, devido à rápida destruição das florestas de restingas pela exploração imobiliária (Baitello et al. 2003, Kropf et al. 2006, Rohwer 1993b) corre alto risco de extinção, estando na lista de espécies ameaçadas da IUCN na categoria "Em Perigo" (EN) (IUCN 2001). Em Mato Grosso do Sul, a espécie é encontrada em uma área restrita em um assentamento rural no município de Corumbá, área degradada devido à exploração florestal. Ocotea aciphylla e Persea willdenovii foram citadas com menor risco pela IUCN (IUCN 2001).

Lauraceae é encontrada em praticamente todas as regiões de Mato Grosso do Sul, no entanto, ocorrem grandes lacunas de conhecimento nas regiões noroeste, sudoeste e nordeste (Fig. 1) do estado em função de sua ampla extensão territorial. Com relação à densidade amostral, a família foi mais intensamente coletada na região central, 


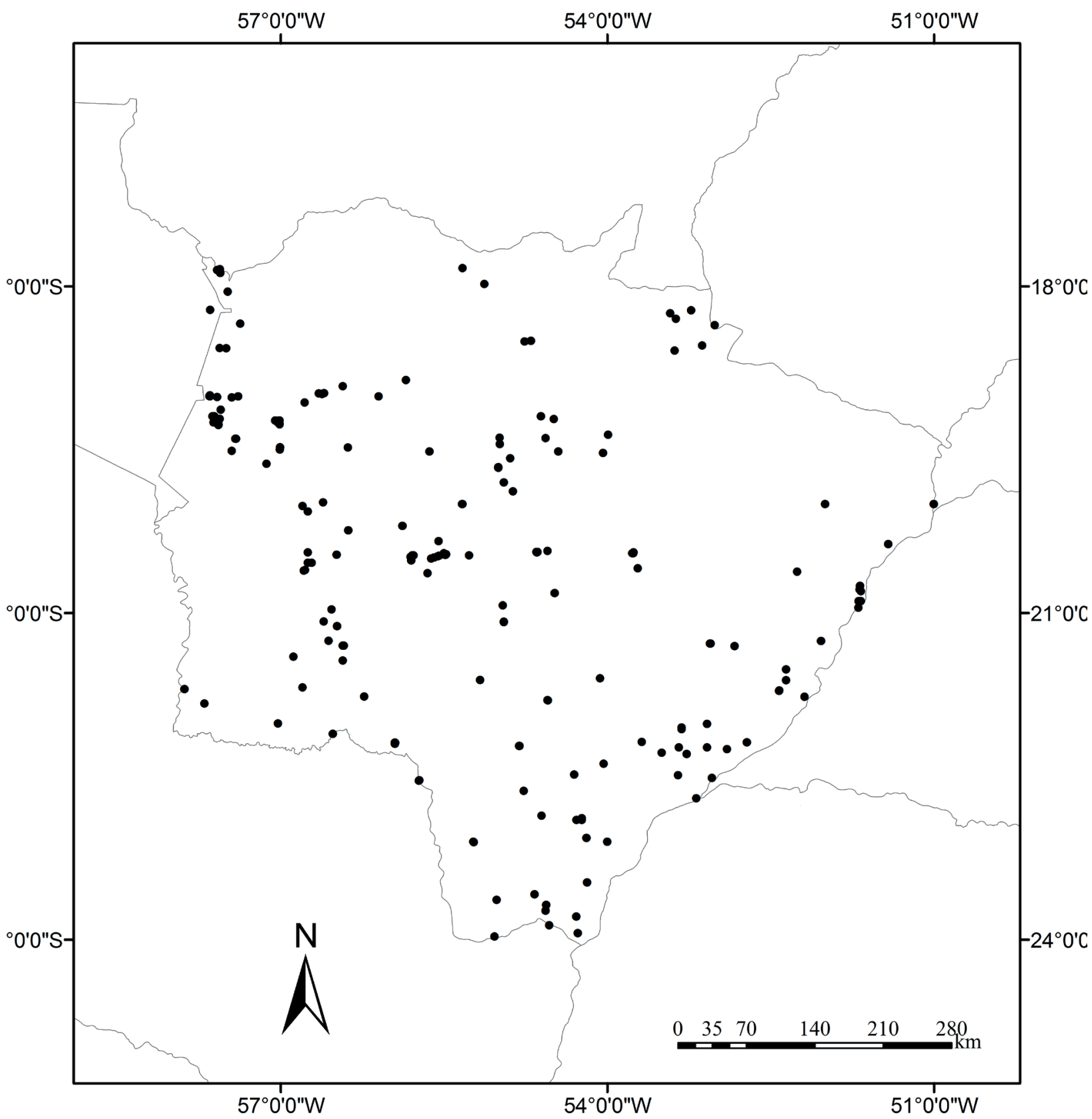

Fig.1. Distribuição geográfica de Lauraceae em Mato Grosso do Sul.

no município de Campo Grande, no centro-oeste do estado nos municípios de Aquidauana, Miranda e Bonito e na porção oeste, no município de Corumbá, um dos maiores do estado. As regiões norte, sudoeste, leste e sul ainda apresentam baixos índices de coletas de Lauraceae (Fig. 2). Este fato, possivelmente, está ligado à presença de importantes instituições locais, tais como a Universidade Federal de Mato Grosso do Sul e da Empresa Brasileira de Pesquisa Agropecuária (Embrapa), o que propicia uma maior disponibilidade de recursos humanos e materiais imprescindíveis aos estudos florísticos e taxonômicos. Coletas intensivas nas regiões sul e sudeste, além de matas ciliares de todo o Mato Grosso do Sul, poderão aumentar consideravelmente o número de gêneros e espécies de Lauraceae conhecidos para o estado. 


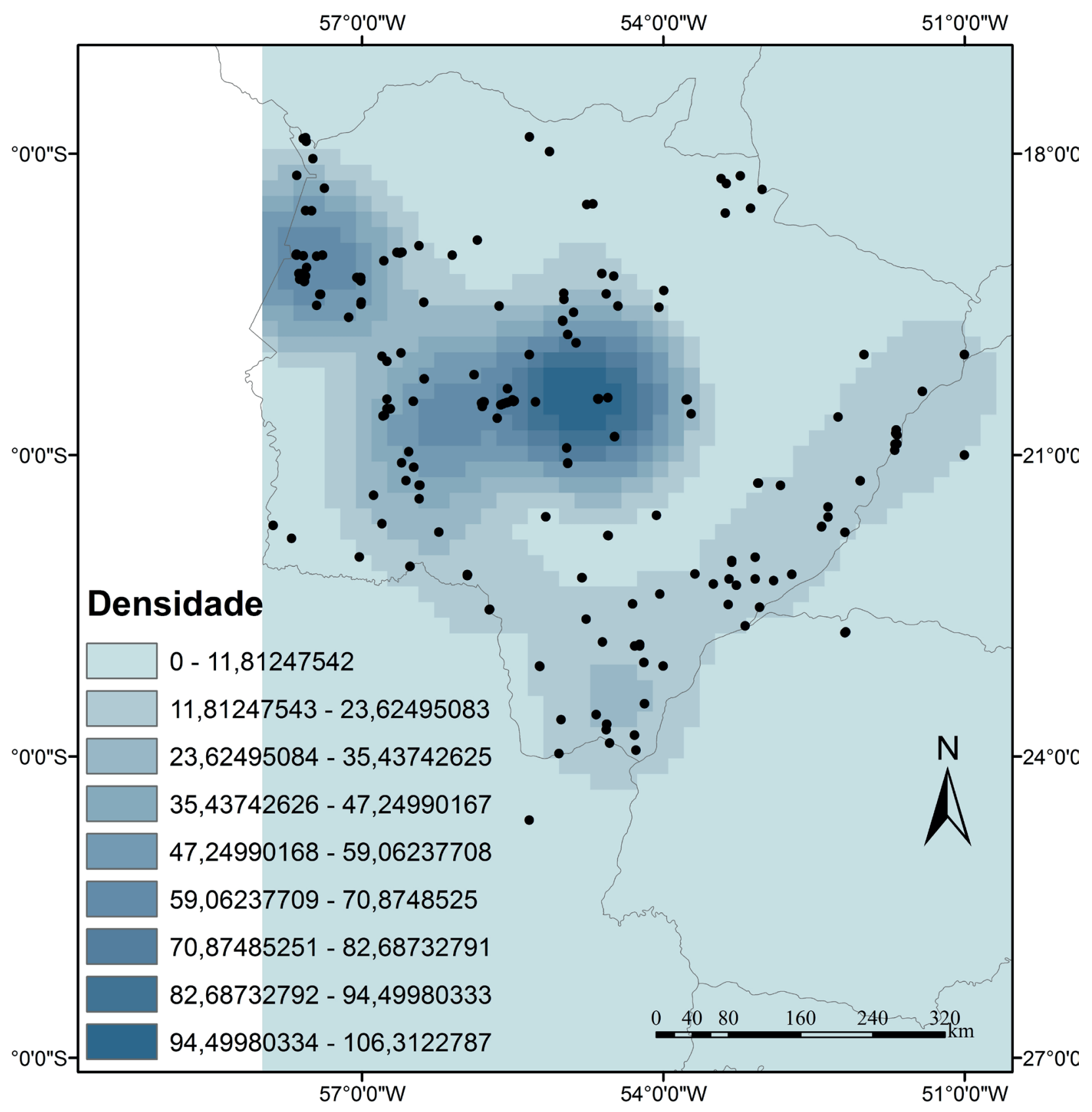

Fig. 2. Localização e densidade de coletas botânicas de Lauraceae em Mato Grosso do Sul.

\section{AGRADECIMENTOS}

Os autores agradecem aos curadores dos herbários por disponibilizarem as informações contidas nas coleções para a realização do trabalho. Os autores agradecem ao também a FUNDECT (Fundação de Apoio ao Desenvolvimento do Ensino, Ciência e Tecnologia do Estado de Mato Grosso do Sul) pelo apoio a pesquisa.

\section{REFERÊNCIAS}

Alves, F.M. 2011. Estudo Taxonômico e Filogenético de Mezilaurus Taub. (Lauraceae) lato sensu e Restabelecimento de Clinostemon Kuhlm. \& A. Samp. Disponível em: www.teses.usp.br/teses/ disponiveis/41/41132/tde-19012012-163504/pt-br.php. Acessado em 12.01.2013.

Alves, F.M. \& Baitello, J.B. 2008. Uma nova espécie de Mezilaurus Taubert (Lauraceae) para a flora brasileira. Acta Botanica Brasilica 22(2):355-358

Alves, F.M. \& Ishii, I.H. 2007. Lauraceae no município de Corumbá, Mato Grosso do Sul, Brasil. Rodriguésia 58(1):179-192.

Alves, F.M. \& Sartori, A.L.B.S. 2009. Nectandra Rol. ex Rottb. (Lauraceae) no Mato Grosso do Sul, Brasil. Acta Botanica Brasilica 23(1):118-129.

Assis, L.C.S., Forzza, R.C. \& Van Der Werff, H. 2005. A família Lauraceae na Reserva Biológica da Represa do Grama, Descoberto, Brasil. Boletim de Botânica 23:113-139.

Baitello, J.B. 2001. Novas espécies de Lauraceae para a flora brasileira. Acta Botanica Brasilica 14(3):445-450.

Baitello, J.B. \& Coe-Teixeira, B. 1987. Flora Fanerogâmica da Reserva do Parque Estadual das Fontes do Ipiranga. Hoehnea 14:63-74. 
Baitello, J.B., Hernández, F.G.L., Moraes, P.L.R., Esteves, R. \& Marcovino, J.R. 2003.

Lauraceae. In Flora Fanerogâmica do Estado de São Paulo (M.G.L. Wanderley, G.J. Shepherd, A.M. Giulietti \& T.S. Melhem, eds.). Editora RiMa, São Paulo, v. 3, p. 149-223.

Beech, E., Rivers, M., Oldfield, S. \& Smith, P.P. 2017. GlobalTreeSearch: The first complete global database of tree species and country distributions. Journal of Sustainable Forestry 36:454-489.

Barroso, G.M., Guimarães, E.F., Ichaso, C.L.F., Costa, C.G. \& Peixoto, A.L. 2002

Sistemática de Angiospermas do Brasil. Universidade Federal de Viçosa, Viçosa. $255 \mathrm{p}$.

Barroso, L.J. 1949. Chave para Determinação de gêneros indígenas e exóticos da família Lauraceae no Brasil. Rodriguésia 12(24):137-146.

Burger, W. 1988. A new genus of Lauraceae from Costa Rica, with comments on problems of generic and specific delimitation within the family. Brittonia 40(3):275-282.

Caiafa, A.N. \& Martins, F.R. 2007. Taxonomic identification, sampling methods, and minimum size of the tree sampled: implications and perspectives for studies in the Brazilian Atlantic Rainforest. Functional Ecosystems and Communities 1(2):95-104

Carvalho, P.E.R. 1994. Espécies florestais brasileiras: recomendações silviculturais, potencialidades e uso da madeira. Embrapa, Brasília. $640 \mathrm{p}$.

Coe-Teixeira, B. 1963. Lauráceas do Estado de São Paulo I. Beilschmiedia, Endlicheria e Aniba. Boletim do Instituto de Botânica de São Paulo $1: 1-29$.

. 1965. Lauráceas do Estado de São Paulo II: Cryptocarya. Arquivos de Botânica do Estado de São Paulo 4(1):1-9.

1975. Espécies Novas de Nectandra (Lauraceae) da Flora do Brasil. Acta Amazônica 5(2):157-171.

1980. Lauráceas do Gênero Ocotea, do Estado de São Paulo. Rodriguésia 52:55-190.

Dubs, B. 1998. The Botany of Mato Grosso. In Checklist of Angiosperms. Betrona-Verlag, Switzerland, série b, n.3, 139 p.

Gentry, A. 1988. Changes in plant community diversity and floristic composition on environmental and geographical gradients. Annals of Missouri Botanical Garden 75:1-34.

Holmgren, P.K., Holmgren, N.H. \& Barnett, L.C. 1990. Index herbariorum: the herbaria of the world. New York Botanical Garden, New York. $693 \mathrm{p}$.

International Union for Conservation of Nature - IUCN. 2001. Red List categories and criteria: Version 3.1. IUCN Species Survival Commission. International Union for Conservation of Nature, Gland, Switzerland and Cambridge, Reino Unido. 36 p.

Kropf, M.S., Quinet, A. \& Andreata, R.H.P. 2006. Lista anotada, distribuição e conservação das espécies de Lauraceae das restingas fluminenses, Brasil. Pesquisas - Botânica 57:161-180.

Kubitzki, K. \& Kurz, H. 1984. Synchronized dichogamy and dioecy in neotropical Lauraceae. Plant Systematics and Evolution 147:253-266.

Lima, R.A.F., Souza, V.C., Dittrich, V.A.O. \& Salino, A. 2012. Composição, diversidade e distribuição geográfica de plantas vasculares de uma Floresta Ombrófila Densa Atlântica do Sudeste do Brasil. Biota Neotropica 12(1):1-10.

Madriñán, S. 2004. Lauraceae. In Flowering plants of the neotropics (N. Smith, S.A.

Mori, A. Henderson, D.W. Stevenson \& S.V. Heald, eds). Princeton University Press, Princeton, p. 204-206.

Ministério do Meio Ambiente - MMA 2012. Lista de espécies ameaçadas da Flora Brasileira. Disponível em: < http://www.mma.gov.br/>. Acessado em: 16.09.2012.

Martinelli, G. \& Martins, E. 2010. Panorama nacional sobre espécies ameaçadas de extinção da flora brasileira. In Diversidade Vegetal Brasileira: conhecimento, conservação e uso. (M.L. Asby, F.D.A. Matos \& I.L. Amaral, orgs). Sociedade Botânica do Brasil, Manaus, p. $592-595$.

Meissner, C.F. 1866. Lauraceae. In Flora Brasiliensis (C.F.P. Martius, ed). Wien Leipzig, Muchen, v.5, pt. 2, p.136-320.

Moraes, P.L.R. 2005. Sinopse das Lauráceas nos estados de Goiás e Tocantins, Brasil. Biota Neotrópica 5(2):1-18.

Moraes, P.L.R. 2006. Taxonomy of Cryptocarya species of Brazil. ABC Taxa Series 1: 1-191.
Pedralli, G.A. 1984. A Família Lauraceae Lindley no Rio Grande do Sul, Brasil: gênero Aiouea Aublet. Iheringia. Série Botânica 32:15-21.

. 1986. A Família Lauraceae Lindley no Rio Grande do Sul, Brasil: Gênero Nectandra Rol. ex Rottb. Iheringia. Série Botânica 35:133149.

1987. Lauráceas: Nectandra - Flora Ilustrada Catarinense. Centro de Tecnologia Mineral, Itajaí. p. 93.

Peixoto, A.L. 2003. Coleções biológicas de apoio ao inventário, uso sustentável e conservação da biodiversidade. Instituto de Pesquisas Jardim Botânico do Rio de Janeiro, Rio de Janeiro, p. 113-125

Pott, A., Pott, V.J., Sciamarelli, A., Sartori, A., Resende, U.M., ScreminDias, E., Jacques, E.L., Aragaki, S., Nakajima, J.N., Romero, R., Cristaldo, A.C.M. \& Damasceno-Junior, G.A. 2006. Inventário de Angiospermas no Complexo Aporé-Sucuriú. In Biodiversidade do Complexo Aporé-Sucuriú: subsídios à conservação e manejo do bioma cerrado (T.C.S. Pagotto \& P.R. Souza, eds.). Universidade Federal de Mato Grosso do Sul, Campo Grande, p. 44-66.

Quinet, A. 2005. Sinopse taxonômica da família Lauraceae no estado do Rio de Janeiro, Brasil. Acta Botanica Brasilica 19:563-572.

2009. Lauraceae. In Plantas da Floresta Atlântica (J.R. Stehmann, R.C. Forzza, A. Salino, M. Sobral, D.P. Costa \& L.H.Y. Kamino, orgs.). Jardim Botânico do Rio de Janeiro, Rio de Janeiro, p. 303-307.

Quinet, A. \& Andreata, R.H.P. 2002. Lauraceae Jussieu na Reserva Ecológica de Macaé de Cima, município de Nova Friburgo, RJ, Brasil. Rodriguésia 53:59-121.

Quinet, A., Baitello, J.B. \& Moraes, P.R.L. 2010. In Catálogo de plantas e fungos do Brasil (R.C. Forzza, J.F.A. Baumgratz, C.E.M. Bicudo, A.A. Carvalho Jr., A. Costa, D.P. Costa, M. Hopkins, P.M. Leitman, L.G. Lohmann, L.C. Maia, G. Martinelli, M. Menezes, M.P. Morim, M.A.N. Coelho, A.L. Peixoto, J.R. Pirani, J. Prado, L.P. Queiroz, V.C. Souza, J.R. Stehmann, L.S. Sylvestre, B.M.T. Walter \& D. Zappi, orgs.). Rio de Andrea Jakobsson Estúdio/ Instituto de Pesquisas Jardim Botânico do Rio de Janeiro, Janeiro, v. 2. p. 1146-1159.

Quinet, A., Baitello, J.B., Moraes, P.L.R., Alves, F.M. \& Assis, L. 2012. Lauraceae. In Lista de Espécies da Flora do Brasil. Jardim Botânico do Rio de Janeiro. Disponível em: http://floradobrasil.jbrj.gov.br/2012/ FB030199. Acessado em 12.05.2012.

Rizzini, C.T. 1979. Tratado de fitogeografia do Brasil: Aspectos ecológicos. Editora da Universidade de São Paulo, São Paulo. 374 p.

Rohwer, J.G. 1993a. Lauraceae. In The families and genera of vascular plants (K. Kubitzki, J.R. Rohwer \& V. Bittrich, eds). Springer-Verlag, Berlin, v. 2, p. 336-391.

. 1993b. Lauraceae: Nectandra. Flora Neotropica 60: 1-332.

Rohwer, J.G., Richter, H.G. \& van der Werff, H. 1991. Two new genera of neotropical Lauraceae and critical remarks on the generic delimitation. Annals of Missouri Botanical Garden 78:388-400.

Sampaio, A.J. 1917. Lauraceas de Matto-Grosso e duas novas espécies da Amazônia. Comissão de Linhas Telegráficas Estratégicas de Mato Grosso ao Amazonas 56 (5):13-15.

Souza, V.C. 2010. Publicações em taxonomia no Brasil: um panorama do momento atual. In Diversidade Vegetal Brasileira: conhecimento, conservação e uso (M.L. Asby, F.D.A. Matos \& I.L. Amaral, orgs). Sociedade Botânica do Brasil, Manaus, p. 335-339.

Souza, V.C. \& Lorenzi, H. 2008. Botânica Sistemática: guia ilustrado para a identificação das famílias de Angiospermas da flora brasileira, baseada em APG II. Nova Odessa, Instituto Plantarum, São Paulo. 704 p.

Thomaz, L.D. \& Monteiro, R. 1997. Composição Florística da Mata Atlântica de encosta da Estação Biológica de Santa Lúcia, Município de Santa Teresa-ES. Boletim do Museu de Biologia Mello Leitão $7: 3-48$.

van der Werff, H. 1987. A Revision of Mezilaurus (Lauraceae). Annals of Missouri Botanical Garden 74:153-182.

1991. A key to the genera of Lauraceae in the new world. Annals of Missouri Botanical Garden 78(2):377-387.

Vattimo-Gil, I. 1956a. O gênero Ocotea Aubl. no sul do Brasil I. Espécies de Santa Catarina e do Paraná. Rodriguésia 18-19(30-31):265-350.

1956b. Nota prévia sobre espécies de Ocotea. Arquivos do Serviço Florestal 10:09-123.

1957. Lauraceae do Estado do Rio de Janeiro: parte I - espécies do Monte Sinai, Governador Portela. Arquivos do Jardim Botânico do Rio de Janeiro 15:115-44. 
1958. Seis novas espécies brasileiras do gênero Ocotea. Arquivos do Serviço Florestal 10:109-123.

1959. Flora da Cidade do Rio de Janeiro (Lauraceae). Rodriguésia 21/22(33-34):157-176.

1966a. Lauraceae do Estado da Guanabara. Rodriguésia 25(37):75113.

. 1966b. Notas sobre o gênero Cryptocarya R. Br. no Brasil (Lauraceae). Rodriguésia 25(37):219-231.

1976. Estudos sobre Ocotea Aubl., Phyllostemonodaphne Kosterm. e Licaria Aubl. (Lauraceae). Rodriguésia 28(41):121-127.

. 1978a. Contribuição ao conhecimento da distribuição geográfica das Lauraceae I. Rodriguésia 29(44):269-306.

1978b. Contribuição ao conhecimento da distribuição geográfica das Lauraceae II. Rodriguésia 30(47):83-103.

1979a. Contribuição ao conhecimento da distribuição geográfica das Lauraceae III. Rodriguésia 31(48):7-57.

1979b. Contribuição ao conhecimento da distribuição geográfica das Lauraceae IV. Rodriguésia 31(49):5-16. 1979c. Contribuição ao conhecimento da distribuição geográfica das Lauraceae V. Novas localidades de ocorrência nos estados do Paraná e Rio Grande do Sul. Rodriguésia 31(50):135-152.

1980a. Contribuição ao conhecimento da distribuição geográfica das Lauraceae VI. Rodriguésia 32:9-32.

1980b. Contribuição ao conhecimento da distribuição geográfica das Lauraceae VII. Rodriguésia 32:351-368.

Vicentini, A., van der Werff, H. \& Nicolau, S. 1999. Lauraceae. In Flora da Reserva Ducke. Guia de Identificação das Plantas Vasculares de uma Floresta de Terra-Firme na Amazônia Central (J.E.L.S. Ribeiro, M.J.G. Hopkins, A. Vicentini, C.A. Sothers, M.A.S. Costa, J.M. Brito, M.A. Solza, L.H.P. Martins, L.G. Lohmann, P.A.C.L. Assunção, E.C. Pereira, C.F. Silva, M.R. Mesquita, L.C. Procópio, orgs). Instituto Nacional de Pesquisas da Amazônia, Manaus, p. 150-179.

Whitmore, T.C. \& Sidiyasa, K. 1986. Composition and structure of a lowland rain forest at Toraut, northern Sulawesi. Kew Bull 41:747756. 